\title{
The Role of Immunometabolism in the Pathogenesis of Systemic Lupus Erythematosus
}

\author{
George Anthony Robinson ${ }^{1,2 t}$, Meredyth G. LI. Wilkinson ${ }^{2,3 t}$ and Chris Wincup ${ }^{1,2 *}$ \\ ${ }^{1}$ Department of Rheumatology, Division of Medicine, University College London, London, United Kingdom, ${ }^{2}$ Centre for \\ Adolescent Rheumatology Versus Arthritis at University College London (UCL), University College London Hospital (UCLH) \\ and Great Ormond Street Hospital (GOSH), University College London, London, United Kingdom, ${ }^{3}$ Department of \\ Rheumatology, University College London Great Ormond Street Institute of Child Health, Infection, Immunity and \\ Inflammation Research and Teaching Department, University College London, London, United Kingdom
}

Systemic lupus erythematosus (SLE) is a chronic autoimmune disorder in which pathogenic abnormalities within both the innate and adaptive immune response have been described. In order to activated, proliferate and maintain this immunological response a drastic upregulation in energy metabolism is required. Recently, a greater understanding of these changes in cellular bioenergetics have provided new insight into the links between immune response and the pathogenesis of a number of diseases, ranging from cancer to diabetes and multiple sclerosis. In this review, we highlight the latest understanding of the role of immunometabolism in SLE with particular focus on the role of abnormal mitochondrial function, lipid metabolism, and mTOR signaling in the immunological phenomenon observed in the SLE. We also consider what implications this has for future therapeutic options in the management of the disease in future.

Keywords: systemic lupus erythematosus (SLE), immunometabolism, mitochondria, lipid metabolism, T cell, B cell, monocyte, autoimmunity

\section{INTRODUCTION}

Systemic lupus erythematosus (SLE) is a chronic autoimmune disorder characterized by the formation of autoantibodies directed against nuclear components. Clinically it may present with a wide array of manifestations and a variety of immunological phenomenon. In spite of recent advances in the management of the disease, therapeutic options remain limited and are often untargeted (1).

The underlying pathogenesis of the disease is poorly understood although abnormal innate and adaptive immune responses have been implicated (2) and is summarized in Figure 1. Observed pathogenic innate responses include dysfunction of macrophages that appear to be defective in removing apoptotic material. It has been suggested that a result of this impaired clearance induces antigenicity to exposed cellular debris including nuclear components $(3,4)$. Macrophages (and their precursors, monocytes) have also been noted to display abnormal polarization in both animal models and in patients with $\operatorname{SLE}(5,6)$. Abnormal neutrophil function has also been observed in the pathogenesis of SLE (7), with recent evidence also implicating the production of neutrophil extracellular traps (NETs) disease development $(8,9)$. In addition, plasmacytoid dendritic cells 


\section{ADAPTIVE}

Autoantibody formation
directed against nuclear component

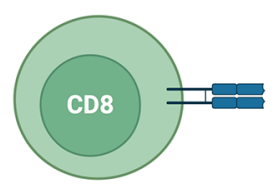

Impaired cytotoxic T cell suppression

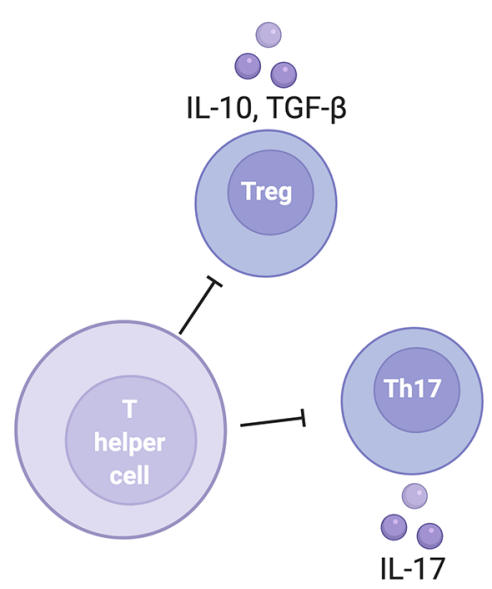

Disbalance in T cell response with reduced suppression from Tregs and increasing numbers of pro-inflammatory Th17 cells

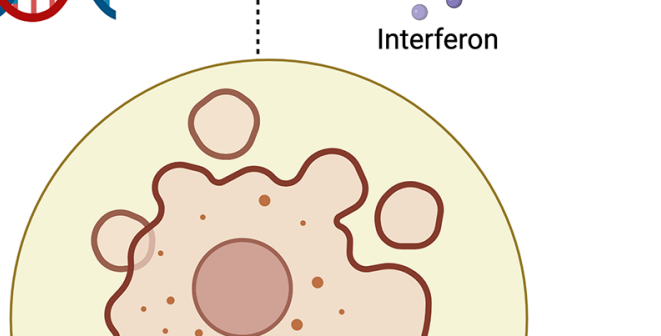

Neutrophils

Neutrophil extracellular traps (NETs) are expelled during innate inflammatory response

FIGURE 1 | A summary of the key immunological abnormalities described in the pathogenesis of systemic lupus erythematosus.

(pDCs) have been identified as another key innate immune driver that has been shown to play a key role in the production in interferon (INF) and generate reactive oxygen species (ROS) $(10,11)$.

The defective clearance of apoptotic matter by dysfunction in the innate immune response is believed to result in a loss of selftolerance and in turn culminates in auto-antibody formation by $B$ cells, which have been noted to show abnormal activation as well as aberrant expression. In turn this results in generation of anti-nuclear antibodies (ANA) and anti-double stranded DNA
(anti-dsDNA) antibodies (4), a hallmark of the disease (12). Furthermore, B cells play a vital role in the development of immune complexes that contain self-antigen, which are deposited within various tissue. The resultant engagement of the $\mathrm{Fc}$ receptor and activation of the complement cascade in turn promotes inflammation $(12,13)$.

$\mathrm{T}$ cells also play a central role in the adaptive immune response and a number of abnormalities have been observed in the pathogenesis of SLE in both propagation and maintenance of the immune response. Regulatory T cells (Tregs) play a vital role 
in maintaining immune homeostasis in health through suppressing a hyperactive immune response. In SLE, an imbalance between pro-inflammatory T helper 17 (Th17) cells and Tregs has been demonstrated as a key contributor to the loss of self-tolerance $(14,15)$. Double negative $\mathrm{T}$ cells derived from patients with SLE have been shown to be an important producer of Interleukin(IL)-17 (16), whilst $\mathrm{CD}^{+}$cytotoxic T cells also demonstrate impaired suppressive function in $\operatorname{SLE}(17,18)$.

The precise mechanism through which this occurs is not known, however, it is felt to involve a combination of genetic factors (19) and environmental triggers (including ultraviolet radiation and possible virus exposure) (20). In addition, given that the disease has a marked female predominance (9:1) there is a growing appreciation of the influence of sex hormones on the autoimmune responses observed (21).

More recently, abnormalities in immunometabolism have been detailed in the defective immune response seen in a variety of disease states including malignancy $(22)$, diabetes $(23,24)$ and multiple sclerosis (25). This has shed new light on the way in which interactions between immunological and metabolic processes may induce the disease state. Immunometabolism also presents a variety of novel therapeutic targets for treatment in the future. In this review, we highlight the latest knowledge in the field of immunometabolism in SLE and describe how this may in turn translate into future clinical care.

\section{ENERGY METABOLISM}

Immune cell activation and proliferation requires significant upregulation in terms of energy metabolism in order to induce and maintain the immunological response. Energy metabolism is dependent on two key pathways to generate adenosine triphosphate (ATP); glycolysis and oxidative phosphorylation (OXPHOS). In health, glycolytic pathways convert glucose to pyruvate and hydrogen ions that are essential for ATP synthesis. In comparison to glycolysis, OXPHOS occurs at the site of the electron transport chain (ETC) on the inner mitochondrial membrane.

\section{MITOCHONDRIAL DYSFUNCTION IN SLE}

Mitochondria are double membrane-bound organelles that generate cellular in the form of ATP, as well as regulating apoptosis. They cannot be replicated by the cell but are formed by binary fission. Each mitochondrion contains a set of circular genome that encode for RNA and proteins which are essential for mitochondrial oxidative phosphorylation. Here we explore the role of mitochondrial dysfunction in the immunopathogenesis of SLE.

\subsection{B Cells (Auto-Antibodies)}

The release of mitochondrial DNA (mtDNA) is a noted marker of acute and chronic disease $(26,27)$. MtDNA activates the innate immune system and can be a target for SLE associated autoantibodies. To identify mitochondrial autoantibodies, a study of 86 SLE patients and 30 healthy controls determined the occurrence of AmtRNA-IgG and Amt-IgM by quantitative ELISA. Both mtRNA immunoglobulins were significantly increased in the SLE patients $(p=0.0002$ and $p=0.0493$, respectively) (28). Antimitochondrial-M2 antibodies (AMAM2) are associated with Primary biliary cirrhosis (PBC) and have been detected at increased levels in subacute cutaneous lupus erythematosus (SCLE) patients (29). In a study of 204 SLE patients, plasma samples were analyzed by ELISA for levels of anti-wMITO. Increased levels correlated to measures of disease activity SLEDAI-2K $(\mathrm{p}<0.0001)$ and SLAM $(\mathrm{p}=0.006)$, antidsDNA $(\mathrm{p}<0.0001)$ and other clinical measures $(30)$. The presence of mitochondrial autoantibodies supports the role of mitochondrial damage in the pathology of SLE. Abnormal mitochondrial function in B cells derived from patients with SLE has more recently been identified. A study of 41 SLE patients and 29 healthy controls found that B cells derived from patients with lupus showed enhanced mitochondrial membrane hyperpolarization, suggesting that these cells are primed for activation. Furthermore, the degree of hyperpolarization correlated with SLEDAI-2K. The authors also noted that glutaminolysis, which generates essential metabolites for OXPHOS, played a key role in the differentiation into plasmablasts (31).

\subsection{T Cells}

$\mathrm{T}$ cell dysfunction in SLE could be attributed to mitochondrial hyperpolarization, reactive oxygen intermediates and reduced levels of ATP (32). Previous studies have demonstrated that T cells are dependent upon glycolytic energy production for the induction of the inflammatory effector response. However, mitochondrial metabolism has also been implicated in the more chronic activation of T cells observed in SLE (33). There is also evidence that in SLE, T cells have increased mitochondrial mass and size both due to defective mitophagy and increased biogenesis (34). Mitochondria contain a reservoir of $\mathrm{Ca}^{2+}$ ions. Increased mitochondrial mass and membrane potential $\left(\uparrow \Delta \psi_{\mathrm{m}}\right)$ in SLE T-cells can increase intracytosolic $\mathrm{Ca}^{2+}$ fluxing when stimulated, in rapamycin treated SLE this was regulated (35). SLE but not healthy control T cells undergo necrosis after CD3/CD28 stimulation due to chronic mitochondrial hyperpolarization (MHP) (36). SLE T cell necrosis can also be caused by increased production of ROS and ATP depletion. Necrotic debris can induce a pro-inflammatory interferon response in plasmacytoid dendritic cells (pDCs) (37). Nitric oxide is released by monocytes which is a driver of MHP. In turn, T cells express intrinsic nitric oxide synthase (iNOS). A meta-analysis showed that there is higher expression of iNOS at both the mRNA and protein level (38). In T cells there is an increased response to IL15 which in turn could contribute to increased mitochondrial biogenesis, though further studies need to be conducted to establish the role of cytokines in mitochondrial dysfunction (39). The status of $\mathrm{T}$ cell metabolic programming can be determined by mitochondrial remodeling as a signaling 
mechanism. This remodeling can change mitochondrial fusion to fission and equally oxidative phosphorylation to aerobic glycolysis. These mechanisms are distinct between effector and memory T cells (40). In SLE T cell oxidative stress is pronounced, increased expression of the mitochondrial protein genes VDAC1 and SOD2 are associated with an increase in mitochondrial mass and oxidative stress $(36,41)$. Other genes associated with mitochondrial dysfunction in SLE are ESRRG, a mitochondrial metabolism regulator, and UCP2, involved in ROS generation and ATP production $(42,43)$. It has been shown that due to oxidative stress, surface glycoprotein $\mathrm{CD} 3 \zeta$ chain is damaged and replaced by FceRI $\gamma$ chain in SLE T cells. The TCR/CD3/ FceRI $\gamma$ complex is up-regulated in effector T-cells and has been shown to be increased in SLE T cells (44).

More recently there is growing evidence to suggest that targeting $\mathrm{T}$ cell metabolism may be a potential therapeutic target for the management of SLE in the future. N-acetylcysteine (NAC) is used clinically as an anti-oxidant therapy and could have a role in targeting oxidative stress in SLE. In a randomized, double-blind, placebo trial of NAC in 36 SLE patients there was significant clinical improvement on $2.4 \mathrm{~g}$ and $4.8 \mathrm{~g}$ dose in terms of Systemic Lupus Erythematosus Disease Activity Index 2000 (SLEDAI-2K) at 1 months ( $p=0.0007), 2$ months $(p=0.0009), 3$ months $(p=0.003)$ and 4 months $(\mathrm{p}=0.0046)$. This study showed that NAC successfully blocks the mammalian target of rapamycin (mTOR) in $\mathrm{T}$ cells (45). Combination treatment with Metformin (a mitochondrial metabolism inhibitor, more commonly used in the treatment of diabetes) and 2-deoxy-d-glucose (2DG) in lupus prone mice has also shown promise, resulting in reduced INF- $\gamma$ production. In addition, mice treated with this therapy showed a reversal of the disease process and reduction in both anti-dsDNA and ANA titers (33). Furthermore, targeting T cell glycolysis has also been demonstrated to specifically reduce the production of follicular helper $\mathrm{T}\left(\mathrm{T}_{\mathrm{FH}}\right)$ cells, which have been implicated in the pathogenesis of SLE (46). Glycolysis has also been investigated as a therapeutic target in another subtype of $\mathrm{CD}^{+} \mathrm{T}$ cells, Th17 cells that predominantly use glycolysis for energy metabolism. In a study of $\mathrm{T}$ cell derived patients with SLE, it was found that by blocking pyruvate dehydrogenase phosphatase catalytic subunit 2 (PDP2), a vital enzyme in the glycolytic pathway, it was possible to limit Th17 differentiation (47). Inhibition of glutaminolysis (a key source of energy for effector $\mathrm{T}$ cells) has also been shown to impact on glycolytic pathways and result in a similar reduction in Th17 differentiation in samples derived from both patients and lupus prone mice, thus suggesting this could also be a potential metabolic therapeutic target in the future Furthermore, the authors also found that inhibition of glutaminolysis reduced Th17 Hypoxia Inducible Factor (HIF)-1 $\alpha$ levels, which plays a central role in Th17 development (48). These studies suggest that through augmentation of $\mathrm{T}$ cell metabolic pathways it may be possible impair abnormal $\mathrm{T}$ cell cytokine production and differentiation.

\subsection{Neutrophils}

Neutrophils taken from SLE patients and healthy control INF primed neutrophils extrude high levels of oxidized mitochondrial nucleoids that act as potent interferogenic complexes, this affect could be due to failed mitophagy. TFAM enables neutrophilderived mtDNA to be internalized and in turn can become a potent $\mathrm{pDC}$ activator. INF/ $\alpha \mathrm{RNP}$ can divert extruded oxidized mtDNA into lysosomes. In turn this drives the formation of ox mtDNA/TFAM complexes which then accumulate in the cytosol and the mitochondria itself. In SLE there are high levels of these oxidized nucleoids in the blood and the neutrophils themselves. In addition, autoantibodies against oxidized mtDNA are present in some SLE patients, proposing ox mtDNA as an autoantigen (49). In SLE and juvenile dermatomyositis (JDM) there are increased levels of neutrophil extracellular traps (NETs) and these have been found to contain mtDNA (50). In SLE, mitochondrial ROS are necessary for NETosis of low density granulocytes (8). Inhibiting mtROS may reduce the INF response in these diseases. In SLE, neutrophils are key to activating the inflammatory mechanism of mtDNA.

\subsection{Monocytes}

A complex study of SLE monocytes showed that excessive INF $\alpha$ in SLE damaged mitochondrial respiration. In the monocytes, SLE compared to healthy control, the results showed increased mitochondrial membrane potential $(\mathrm{p}<0.0005)$, PINK1 mRNA $(\mathrm{p}<0.005)$, mtDNA content $(\mathrm{p}<0.005)$ and JC1 aggregates $(\mathrm{p}<0.05)$. These results were re-produced when healthy donor monocytes were cultured with INF $\alpha$ for $18 \mathrm{hrs}$ (51). This delineates the cyclical relationship of INF $\alpha$ with mitochondrial dysfunction.

Across the innate and adaptive immune cells there is strong evidence that mitochondrial dysfunction plays an important role in SLE immunopathogenesis. Therefore, is an important therapeutic target to consider.

\section{ABNORMAL mTOR SIGNALING IN SLE}

Another important group of substrates involved immune cell metabolism are proteins, peptides and amino acids. There is now a growing appreciation of their role in autoimmunity, in particular in relation to their effects on $\mathrm{T}$ cell differentiation and function. This relies upon the activation of the serine-theonine protein kinase, mTOR, which exists in two separate complexes known as mTORC1 and mTORC2 (52). Furthermore, mTOR is essential in the maintenance of immune cell homeostasis through its roles in inducing metabolic signals that in turn drive cell growth, activation, proliferation and survival (52-55).

In health, mTORC1 plays a key role in the suppressive function of Tregs, a mechanism that has been demonstrated to be abnormal in many autoimmune conditions (55-57). In SLE, abnormalities within mTOR pathways have been shown to induce immune cell differentiation and proliferation, secretion of pro-inflammatory cytokines and increased ROS production (58). Previous studies have demonstrated the role of mTORC1 activation in $\mathrm{CD}^{+} \mathrm{T}$ cells derived from patients with SLE (53) and has suggested that this may be due to mitochondrial dysfunction (45). More specifically, mTOR abnormalities have been reported to alter the balance between Th17 T cells and 
Tregs to the extent that it promotes a state autoimmunity (59). This increase in mTORC1 activity has been demonstrated following increased glycolysis and also associates with reduced levels of autophagy (54), which is impaired in the pathogenesis of SLE (60).

There is growing evidence that targeting mTOR may also be an effective treatment in the management of SLE clinically. Sirolimus (Rapamycin), an immunosuppressive agent used in preventing graft rejection in solid organ transplantation and known mTORC1 inhibitor, has already been studies as a potential treatment for SLE. Inhibition of mTOR with Rapamycin has already been shown to reduced INF production by monocytes derived from patients with SLE in vitro (61). In a previous open-label study in 43 patients with active SLE found that following 12 months of treatment with Sirolimus, disease activity was significantly reduced, and concurrent steroid dose was also significantly lower following one year of treatment. Immunologically, Sirolimus was also noted to induce increased numbers of Tregs, which suggests a recovery immune homeostasis with the treatment. Furthermore, T cell produced IL-4 and IL-17 levels were also significantly lower following treatment (62). Although there is a lack of large randomized, placebo-controlled trials of the drug in lupus, a recent metaanalysis of nine studies containing a total of 145 patients concluded that Sirolimus showed promise as a treatment option. It was suggested that the drug was well tolerated (although hematological and mucocutaneous adverse events were the most frequently reported) (63). Inhibition of mTOR with Sirolimus additionally was associated with higher rates of dyslipidemia, which is important given the growing evidence for abnormalities in lipid metabolism in SLE.

\section{LIPID METABOLISM IN SLE}

The metabolism of lipids is a fundamental process used by immune cells for different energy demands, cell signaling and function. Lipids serve as precursors for bioactive metabolites and components of cellular membranes, which have both direct and indirect regulatory implications for signal transduction, gene regulation and cellular activation. Immune cell subsets have different metabolic demands for lipids, such as mitochondrial beta-oxidation of lipids for anti-inflammatory functions in regulatory $\mathrm{T}$ cells, against a higher dependency on glycolytic pathways for growth and proliferation in effector $\mathrm{T}$ cells (64). Dysregulated lipid metabolism has been heavily implicated in SLE at both the systemic and cellular level and both have been described in the context of cardiovascular comorbidities.

\subsection{Lipid Metabolism and Cardiovascular Disease in SLE}

Patients with SLE have an increased risk of developing cardiovascular disease (CVD) beyond traditional risk factors and CVD is a leading cause of mortality for patients (65). This CVD risk is largely due to dyslipidemia (altered lipid metabolism), a common feature of SLE (66). Dyslipidemia can accelerate atherosclerosis, the lipid build-up and chronic inflammation of the large arteries (67). This involves an imbalance between atherogenic low and very low density lipoproteins (LDL and VLDL), and atheroprotective high density lipoproteins (HDL) known to transport lipids too and away from atherosclerotic plaques respectively. Dyslipidemia in SLE includes both elevated LDL and reduced HDL $(66,68-70)$ which, along with chronic inflammation, accelerates atherosclerotic processes.

\subsection{Lipid Metabolism in Immune Cell Function in SLE}

Lipoprotein metabolism can also influence immune cell function and inflammation in SLE (71). It is well established that innate immune cells, including macrophages, take up oxidized (ox)LDL particles via scavenger receptors in atherosclerotic plaques, leading to lipid saturation, pro-inflammatory cytokine production, and recruitment of other inflammatory cells (72). This process could be exacerbated in SLE due to the increased circulating levels of LDL, thus, increasing atherosclerosis progression. In addition, macrophage function is likely to be altered via direct lipid activation of the nuclear liver-Xreceptors (LXRs), which regulate cellular cholesterol levels and immune functions through transcriptional changes, such as those involved in IL-23 and IL-17 production and phagocytic pathways (73). The direct effect of a hyperlipidemic environment on the $\mathrm{T}$ cell inflammatory profile in SLE has also been investigated $(74,75)$ and oxLDL has been shown to increase $\mathrm{T}$ cell activation indirectly through monocyte uptake (76). T cells are key for the adaptive immune system and upon activation, $\mathrm{T}$ cells proliferate, migrate to inflamed sites, such as atherosclerotic plaques, and acquire functions that mediate the immune response (77). The $\mathrm{T}$ cell plasma membrane (PM) is made up abundantly of lipids, such as cholesterol and phospholipids, and proteins, both of which are essential to facilitate cellular signaling for inflammatory outcomes such as cytokine production and proliferation (78). Patients with SLE and other autoimmune diseases have altered $\mathrm{T}$ cell membrane cholesterol and glycosphingolipid levels $(79,80)$. This alters the composition of signaling platforms called lipid rafts, where $\mathrm{T}$ cell receptors provide stimulatory signals to control cellular function and inflammation $(81,82)$. This is partly due differences in the expression of genes responsible for lipid metabolism in SLE (71), however, this could also be due altered cellular altered uptake of cholesterol from LDL/VLDL and efflux of cholesterol to HDL; this process has been speculated in pathogenic mechanisms of multiple sclerosis (83). Altered lipid rafts have also been described in the context of dysfunctional B cell signaling in SLE (84). Altered lymphocyte function through dyslipidemia in SLE is also likely to be mediated through LXRs $(82,85,86)$. Together, research strongly suggest that lipid metabolism could be targeted therapeutically to control cellular functions and inflammation, highlighting the need for a better use of lipid modification strategies in SLE. 


\subsection{Lipid Metabolism as a Therapeutic Target}

Some conventional therapeutics currently being used to treat SLE have shown beneficial effects on lipids, including hydroxychloroquine on LDL lowering (87). Despite this, deaths associated with cardiovascular comorbidities are still high (65, 88) suggesting that additional, more specific lipid modifying therapies are in demand for patients with SLE. Despite statin trials in SLE showing mixed results regarding cardiovascular outcome measures (89-91), therapeutically lowering circulating lipid levels has been shown to improve autoimmune disease symptoms (92) and using these therapies to directly modify lipid rafts in vitro has also been shown to normalize signaling in $\mathrm{T}$ cells from SLE patients $(80,93)$.

Taken together, differences in lipid metabolism in patients with SLE contributes to disease pathogenesis, inflammation and CVD risk through atherosclerosis. Therapeutic intervention with lipid modifying drugs already approved for use worldwide, such as statins, could be promising strategies to control atherosclerosis and inflammation in SLE. The success of future clinical trials and the therapeutic application of these treatments is likely to be dependent on correct patient stratification. Reducing CVD risk in SLE patents from a young age will be a huge breakthrough for long term patient outcomes and quality of life.

\section{CONCLUSIONS}

The field of immunometabolism has enhanced our understanding of the key changes in cellular homeostasis and how this can result in autoimmune conditions including SLE. Observed mitochondrial dysfunction has implications for immune cell energy metabolism and also ROS generation. Abnormalities within mTOR signaling may induce promote immune cell differentiation and proliferation, whilst also stimulating pro-inflammatory cytokine production. Lipid metabolism has been shown to potentially play a role in immune cell signaling. Figure 2 summarizes the key changes in immunometabolism observed in SLE to date.

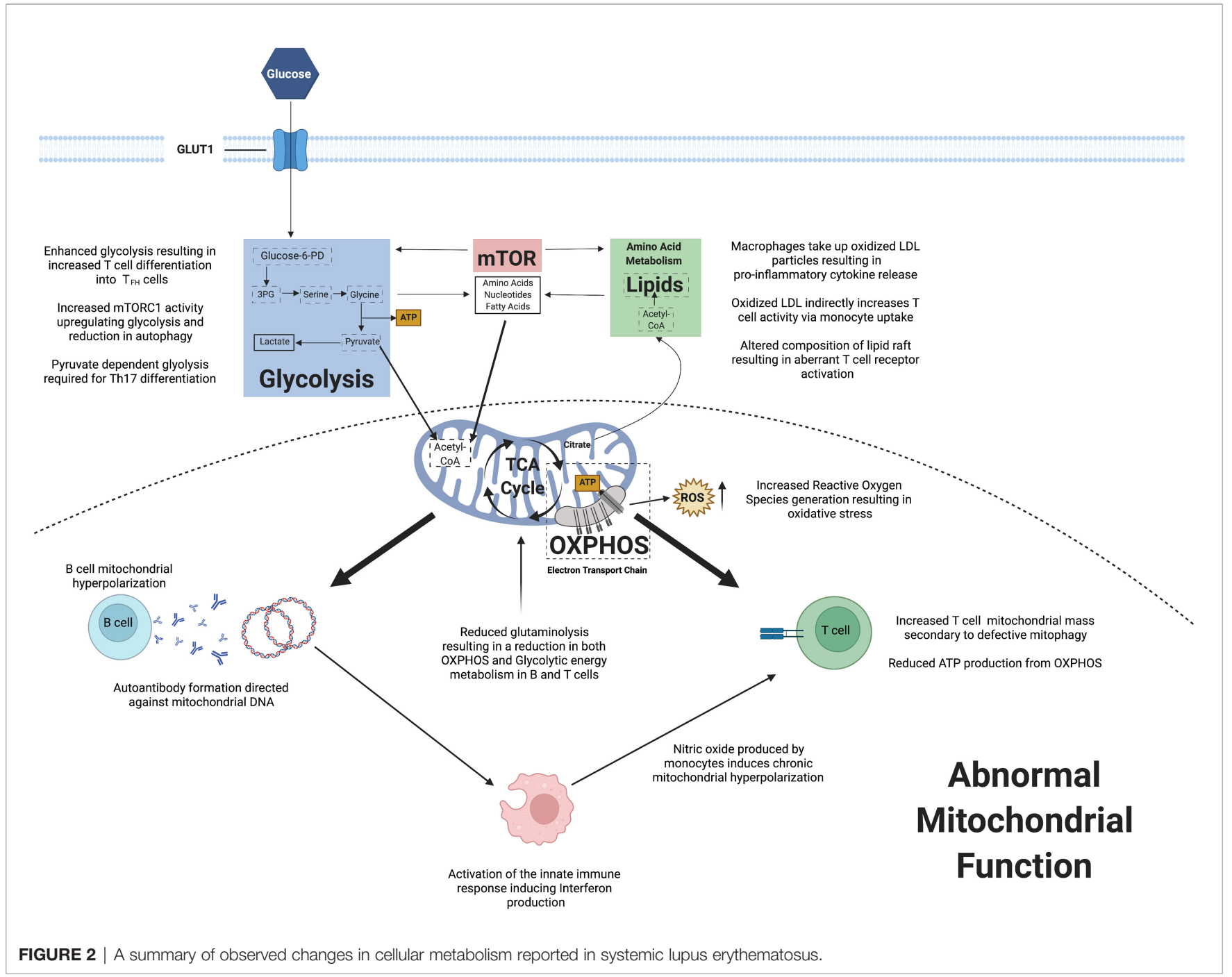


In conclusion, our understanding of immunometabolism in SLE is rapidly increasing and main soon translate to newer agents being developed specifically to restore immune cell homeostasis in the disease.

\section{AUTHOR CONTRIBUTIONS}

MW was responsible for writing Section 3 (Mitochondrial dysfunction in SLE). GR was responsible for writing Section 4 (Lipid metabolism in SLE). CW was responsible for the remaining sections and editing the final manuscript. All authors contributed to the article and approved the submitted version.

\section{REFERENCES}

1. Bakshi J, Segura BT, Wincup C, Rahman A. Unmet Needs in the Pathogenesis and Treatment of Systemic Lupus Erythematosus. Clin Rev Allergy Immunol (2018) 55(3):352-67. doi: 10.1007/s12016-017-8640-5

2. Katsiari CG, Liossis SN, Sfikakis PP. The Pathophysiologic Role of Monocytes and Macrophages in Systemic Lupus Erythematosus: A Reappraisal. Semin Arthritis Rheum (2010) 39(6):491-503. doi: 10.1016/j.semarthrit.2008.11.002

3. Bijl M, Reefman E, Horst G, Limburg PC, Kallenberg CG. Reduced Uptake of Apoptotic Cells by Macrophages in Systemic Lupus Erythematosus: Correlates With Decreased Serum Levels of Complement. Ann Rheumatic Dis (2006) 65(1):57-63. doi: 10.1136/ard.2005.035733

4. Tas SW, Quartier P, Botto M, Fossati-Jimack L. Macrophages From Patients With SLE and Rheumatoid Arthritis Have Defective Adhesion In Vitro, While Only SLE Macrophages Have Impaired Uptake of Apoptotic Cells. Ann rheumatic Dis (2006) 65(2):216-21. doi: 10.1136/ard.2005.037143

5. Mohammadi S, Saghaeian-Jazi M, Sedighi S, Memarian A. Immunomodulation in Systemic Lupus Erythematosus: Induction of M2 Population in Monocyte-Derived Macrophages by Pioglitazone. Lupus (2017) 26(12):1318-27. doi: 10.1177/0961203317701842

6. Li F, Yang Y, Zhu X, Huang L, Xu J. Macrophage Polarization Modulates Development of Systemic Lupus Erythematosus. Cell Physiol Biochem Int J Exp Cell physiol biochem Pharmacol (2015) 37(4):1279-88. doi: 10.1159/ 000430251

7. Chapman EA, Lyon M, Simpson D, Mason D, Beynon RJ, Moots RJ, et al. Caught in a Trap? Proteomic Analysis of Neutrophil Extracellular Traps in Rheumatoid Arthritis and Systemic Lupus Erythematosus. Front Immunol (2019) 10:423. doi: 10.3389/fimmu.2019.00423

8. Lood C, Blanco LP, Purmalek MM, Carmona-Rivera C, De Ravin SS, Smith CK, et al. Neutrophil Extracellular Traps Enriched in Oxidized Mitochondrial DNA are Interferogenic and Contribute to Lupus-Like Disease. Nat Med (2016) 22(2):146-53. doi: 10.1038/nm.4027

9. Knight JS, Kaplan MJ. Lupus Neutrophils: 'NET' Gain in Understanding Lupus Pathogenesis. Curr Opin Rheumatol (2012) 24(5):441-50. doi: 10.1097/ BOR.0b013e3283546703

10. Eloranta ML, Lovgren T, Finke D, Mathsson L, Ronnelid J, Kastner B, et al. Regulation of the Interferon-Alpha Production Induced by RNA-Containing Immune Complexes in Plasmacytoid Dendritic Cells. Arthritis rheumatism (2009) 60(8):2418-27. doi: 10.1002/art.24686

11. Menon M, Blair PA, Isenberg DA, Mauri C. A Regulatory Feedback Between Plasmacytoid Dendritic Cells and Regulatory B Cells Is Aberrant in Systemic Lupus Erythematosus. Immunity (2016) 44(3):683-97. doi: 10.1016/ j.immuni.2016.02.012

12. Pickering MC, Botto M, Taylor PR, Lachmann PJ, Walport MJ. Systemic Lupus Erythematosus, Complement Deficiency, and Apoptosis. Adv Immunol (2000) 76:227-324. doi: 10.1016/S0065-2776(01)76021-X

13. Feng Y, Yang M, Wu H, Lu Q. The Pathological Role of B Cells in Systemic Lupus Erythematosus: From Basic Research to Clinical. Autoimmunity (2019) 53(2):56-64. doi: 10.1080/08916934.2019.1700232

\section{FUNDING}

GR is funded by a combined Lupus UK and the Rosetrees Trust grant (ref M409). MW is funded by Cure JM and GOS BRC. CW is funded by Versus Arthritis (ref 21992 and 22600) and Lupus UK.

\section{ACKNOWLEDGMENTS}

CW would like to acknowledge the support he receives from Versus Arthritis and LUPUS UK. Figures are created with Biorender.com.

14. Álvarez-Rodríguez L, Martínez-Taboada V, Calvo-Alén J, Beares I, Villa I, López-Hoyos M. Altered Th17/Treg Ratio in Peripheral Blood of Systemic Lupus Erythematosus But Not Primary Antiphospholipid Syndrome. Front Immunol (2019) 10:391. doi: 10.3389/fimmu.2019.00391

15. Yang J, Yang X, Zou H, Chu Y, Li M. Recovery of the Immune Balance Between Th17 and Regulatory T Cells as a Treatment for Systemic Lupus Erythematosus. Rheumatol (Oxf Engl) (2011) 50(8):1366-72. doi: 10.1093/ rheumatology/ker116

16. Li H, Adamopoulos IE, Moulton VR, Stillman IE, Herbert Z, Moon JJ, et al. Systemic Lupus Erythematosus Favors the Generation of IL-17 Producing Double Negative T Cells. Nat Commun (2020) 11(1):2859. doi: 10.1038/ s41467-020-16636-4

17. Filaci G, Bacilieri S, Fravega M, Monetti M, Contini P, Ghio M, et al. Impairment of CD8+ T Suppressor Cell Function in Patients With Active Systemic Lupus Erythematosus. J Immunol (2001) 166(10):6452-7. doi: 10.4049/jimmunol.166.10.6452

18. Dinesh RK, Skaggs BJ, La Cava A, Hahn BH, Singh RP. CD8+ Tregs in Lupus, Autoimmunity, and Beyond. Autoimmun Rev (2010) 9(8):560-8. doi: 10.1016/j.autrev.2010.03.006

19. Song K, Liu L, Zhang X, Chen X. An Update on Genetic Susceptibility in Lupus Nephritis. Clin Immunol (2019) 210:108272. doi: 10.1016/ j.clim.2019.108272

20. Stannard JN, Kahlenberg JM. Cutaneous Lupus Erythematosus: Updates on Pathogenesis and Associations With Systemic Lupus. Curr Opin Rheumatol (2016) 28(5):453-9. doi: 10.1097/BOR.0000000000000308

21. Jones BG, Penkert RR, Surman SL, Sealy RE, Pelletier S, Xu B, et al. Matters of Life and Death: How Estrogen and Estrogen Receptor Binding to the Immunoglobulin Heavy Chain Locus may Influence Outcomes of Infection, Allergy, and Autoimmune Disease. Cell Immunol (2019) 346:103996. doi: 10.1016/j.cellimm.2019.103996

22. Dyck L, Lynch L. Cancer, Obesity and Immunometabolism - Connecting the Dots. Cancer Lett (2018) 417:11-20. doi: 10.1016/j.canlet.2017.12.019

23. Kohlgruber AC, LaMarche NM, Lynch L. Adipose Tissue at the Nexus of Systemic and Cellular Immunometabolism. Semin Immunol (2016) 28 (5):431-40. doi: 10.1016/j.smim.2016.09.005

24. Lenin R, Sankaramoorthy A, Mohan V, Balasubramanyam M. Altered Immunometabolism at the Interface of Increased Endoplasmic Reticulum (ER) Stress in Patients With Type 2 Diabetes. J Leukocyte Biol (2015) 98 (4):615-22. doi: 10.1189/jlb.3A1214-609R

25. Luu M, Pautz S, Kohl V, Singh R, Romero R, Lucas S, et al. The Short-Chain Fatty Acid Pentanoate Suppresses Autoimmunity by Modulating the Metabolic-Epigenetic Crosstalk in Lymphocytes. Nat Commun (2019) 10 (1):760. doi: 10.1038/s41467-019-08711-2

26. Hajizadeh S, DeGroot J, TeKoppele JM, Tarkowski A, Collins LV. Extracellular Mitochondrial DNA and Oxidatively Damaged DNA in Synovial Fluid of Patients With Rheumatoid Arthritis. Arthritis Res Ther (2003) 5(5):R234-40. doi: 10.1186/ar787

27. Liu J, Zou Y, Tang Y, Xi M, Xie L, Zhang Q, et al. Circulating Cell-Free Mitochondrial Deoxyribonucleic Acid is Increased in Coronary Heart Disease 
Patients With Diabetes Mellitus. J Diabetes Investig (2016) 7(1):109-14. doi: $10.1111 /$ jdi. 12366

28. Becker Y, Marcoux G, Allaeys I, Julien AS, Loignon RC, Benk-Fortin H, et al. Autoantibodies in Systemic Lupus Erythematosus Target Mitochondrial RNA. Front Immunol (2019) 10:1026. doi: 10.3389/fimmu.2019.01026

29. Pelka K, Stec-Polak M, Wojas-Pelc A, Pastuszczak M. Prevalence of Antimitochondrial Antibodies in Subacute Cutaneous Lupus Erythematosus. Int J Dermatol (2021) 60(1):88-92. doi: 10.1111/ijd.15225

30. Pisetsky DS, Spencer DM, Mobarrez F, Fuzzi E, Gunnarsson I, Svenungsson E. The Binding of SLE Autoantibodies to Mitochondria. Clin Immunol (2020) 212:108349. doi: 10.1016/j.clim.2020.108349

31. Sumikawa MH, Iwata S, Zhang M, Miyata H, Ueno M, Todoroki Y, et al. An Enhanced Mitochondrial Function Through Glutamine Metabolism in Plasmablast Differentiation in Systemic Lupus Erythematosus. Rheumatol (Oxf Engl) (2021). doi: 10.1093/rheumatology/keab824

32. Gergely P Jr, Grossman C, Niland B, Puskas F, Neupane H, Allam F, et al. Mitochondrial Hyperpolarization and ATP Depletion in Patients With Systemic Lupus Erythematosus. Arthritis rheumatism (2002) 46(1):175-90. doi: 10.1002/1529-0131(200201)46:1<175::AID-ART10015>3.0.CO;2-H

33. Yin Y, Choi SC, Xu Z, Perry DJ, Seay H, Croker BP, et al. Normalization of CD4+ T Cell Metabolism Reverses Lupus. Sci Trans Med (2015) 7 (274):274ra18. doi: 10.1126/scitranslmed.aaa0835

34. Caza TN, Talaber G, Perl A. Metabolic Regulation of Organelle Homeostasis in Lupus T Cells. Clin Immunol (2012) 144(3):200-13. doi: 10.1016/ j.clim.2012.07.001

35. Fernandez D, Bonilla E, Mirza N, Niland B, Perl A. Rapamycin Reduces Disease Activity and Normalizes T Cell Activation-Induced Calcium Fluxing in Patients With Systemic Lupus Erythematosus. Arthritis rheumatism (2006) 54(9):2983-8. doi: 10.1002/art.22085

36. Nagy G, Koncz A, Perl A. T Cell Activation-Induced Mitochondrial Hyperpolarization is Mediated by Ca2+- and Redox-Dependent Production of Nitric Oxide. J Immunol (2003) 171(10):5188-97. doi: 10.4049/ jimmunol.171.10.5188

37. Kis-Toth K, Tsokos GC. Dendritic Cell Function in Lupus: Independent Contributors or Victims of Aberrant Immune Regulation. Autoimmunity (2010) 43(2):121-30. doi: 10.3109/08916930903214041

38. Pan L, Yang S, Wang J, Xu M, Wang S, Yi H. Inducible Nitric Oxide Synthase and Systemic Lupus Erythematosus: A Systematic Review and Meta-Analysis. BMC Immunol (2020) 21(1):6. doi: 10.1186/s12865-020-0335-7

39. Baranda L, de la Fuente H, Layseca-Espinosa E, Portales-Pérez D, NiñoMoreno P, Valencia-Pacheco G, et al. IL-15 and IL-15R in Leucocytes From Patients With Systemic Lupus Erythematosus. Rheumatol (Oxf Engl) (2005) 44(12):1507-13. doi: 10.1093/rheumatology/kei083

40. Buck MD, O'Sullivan D, Klein Geltink RI, Curtis JD, Chang CH, Sanin DE, et al. Mitochondrial Dynamics Controls T Cell Fate Through Metabolic Programming. Cell (2016) 166(1):63-76. doi: 10.1016/j.cell.2016.05.035

41. Fernandez DR, Telarico T, Bonilla E, Li Q, Banerjee S, Middleton FA, et al. Activation of Mammalian Target of Rapamycin Controls the Loss of TCRzeta in Lupus T Cells Through HRES-1/Rab4-Regulated Lysosomal Degradation. J Immunol (2009) 182(4):2063-73. doi: 10.4049/jimmunol.0803600

42. Yu X, Wieczorek S, Franke A, Yin H, Pierer M, Sina C, et al. Association of UCP2 -866 G/A Polymorphism With Chronic Inflammatory Diseases. Genes Immun (2009) 10(6):601-5. doi: 10.1038/gene.2009.29

43. Huss JM, Garbacz WG, Xie W. Constitutive Activities of Estrogen-Related Receptors: Transcriptional Regulation of Metabolism by the ERR Pathways in Health and Disease. Biochim Biophys Acta (2015) 1852(9):1912-27. doi: 10.1016/j.bbadis.2015.06.016

44. Krishnan S, Warke VG, Nambiar MP, Tsokos GC, Farber DL. The FcR Gamma Subunit and Syk Kinase Replace the CD3 Zeta-Chain and ZAP-70 Kinase in the TCR Signaling Complex of Human Effector CD4 T Cells. J Immunol (2003) 170(8):4189-95. doi: 10.4049/jimmunol.170.8.4189

45. Lai ZW, Hanczko R, Bonilla E, Caza TN, Clair B, Bartos A, et al. NAcetylcysteine Reduces Disease Activity by Blocking Mammalian Target of Rapamycin in T Cells From Systemic Lupus Erythematosus Patients: A Randomized, Double-Blind, Placebo-Controlled Trial. Arthritis Rheumatism (2012) 64(9):2937-46. doi: 10.1002/art.34502

46. Choi SC, Titov AA, Abboud G, Seay HR, Brusko TM, Roopenian DC, et al. Inhibition of Glucose Metabolism Selectively Targets Autoreactive Follicular
Helper T Cells. Nat Commun (2018) 9(1):4369. doi: 10.1038/s41467-01806686-0

47. Kono M, Yoshida N, Maeda K, Skinner NE, Pan W, Kyttaris VC, et al. Pyruvate Dehydrogenase Phosphatase Catalytic Subunit 2 Limits Th17 Differentiation. Proc Natl Acad Sci USA (2018) 115(37):9288-93. doi: 10.1073/pnas. 1805717115

48. Kono M, Yoshida N, Maeda K, Suárez-Fueyo A, Kyttaris VC, Tsokos GC. Glutaminase 1 Inhibition Reduces Glycolysis and Ameliorates Lupus-Like Disease in MRL/lpr Mice and Experimental Autoimmune Encephalomyelitis. Arthritis Rheumatol (Hoboken NJ) (2019) 71(11):1869-78. doi: 10.1002/ art.41019

49. Caielli S, Athale S, Domic B, Murat E, Chandra M, Banchereau R, et al. Oxidized Mitochondrial Nucleoids Released by Neutrophils Drive Type I Interferon Production in Human Lupus. J Exp Med (2016) 213(5):697-713. doi: 10.1084/jem.20151876

50. Duvvuri B, Pachman LM, Morgan G, Khojah AM, Klein-Gitelman M, Curran ML, et al. Neutrophil Extracellular Traps in Tissue and Periphery in Juvenile Dermatomyositis. Arthritis Rheumatol (Hoboken NJ) (2020) 72(2):348-58. doi: $10.1002 /$ art.41078

51. Gkirtzimanaki K, Kabrani E, Nikoleri D, Polyzos A, Blanas A, Sidiropoulos P, et al. Ifn $\alpha$ Impairs Autophagic Degradation of mtDNA Promoting Autoreactivity of SLE Monocytes in a STING-Dependent Fashion. Cell Rep (2018) 25(4):921-33.e5. doi: 10.1016/j.celrep.2018.09.001

52. Perl A. Activatiion of mTOR Mechanistic Target of Rapamycin in Rheumatic Diseases. Nat Rev Rheumtol (2016) 12(3):169-82. doi: 10.1038/nrrheum.2015.172

53. Li W SR, Titov AA, Choi SC, Morel L. Metabolic Factors That Contribute to Lupus Pathogenesis. Crit Rev Immunol (2016) 36(1):75-98. doi: 10.1615/ CritRevImmunol.2016017164

54. Laurence M. Immunometabolism in Systemic Lupus Erythematosus. Nat Rev Rheumatol (2017) 13(5):280-90. doi: 10.1038/nrrheum.2017.43

55. Hu Z, Kai Y, Caryn C, Geoffrey N, Peter V, Hongbo C. Mtorc1 Couples Immune Signals and Metabolic Programming to Establish Treg-Cell Function. Nature (2013) 499(7459):485. doi: 10.1038/nature12297

56. Mizui M, Tsokos GC. Targeting Regulatory T Cells to Treat Patients With Systemic Lupus Erythematosus. Front Immunol (2018) 9:786. doi: 10.3389/ fimmu.2018.00786

57. Vilà L, Roglans N, Baena M, Barroso E, Alegret M, Merlos M, et al. Metabolic Alterations and Increased Liver mTOR Expression Precede the Development of Autoimmune Disease in a Murine Model of Lupus Erythematosus. PloS One (2012) 7(12):e51118. doi: 10.1371/journal.pone.0051118

58. He J, Ma J, Ren B, Liu A. Advances in Systemic Lupus Erythematosus Pathogenesis via mTOR Signaling Pathway. Semin Arthritis Rheum (2020) 50(2):314-20. doi: 10.1016/j.semarthrit.2019.09.022

59. Shan J, Jin H, Xu Y. T Cell Metabolism: A New Perspective on Th17/Treg Cell Imbalance in Systemic Lupus Erythematosus. Front Immunol (2020) 11:1027. doi: 10.3389/fimmu.2020.01027

60. Kato H, Perl A. Blockade of Treg Cell Differentiation and Function by the Interleukin-21-Mechanistic Target of Rapamycin Axis Via Suppression of Autophagy in Patients With Systemic Lupus Erythematosus. Arthritis Rheumatol (Hoboken NJ) (2018) 70(3):427-38. doi: 10.1002/art.40380

61. Murayama G, Chiba A, Kuga T, Makiyama A, Yamaji K, Tamura N, et al. Inhibition of mTOR Suppresses Ifn $\alpha$ Production and the STING Pathway in Monocytes From Systemic Lupus Erythematosus Patients. Rheumatol (Oxford England) (2020) 59(10):2992-3002. doi: 10.1093/rheumatology/keaa060

62. Lai ZW, Kelly R, Winans T, Marchena I, Shadakshari A, Yu J, et al. Sirolimus in Patients With Clinically Active Systemic Lupus Erythematosus Resistant to, or Intolerant of, Conventional Medications: A Single-Arm, Open-Label, Phase 1/2 Trial. Lancet (London England) (2018) 391(10126):1186-96. doi: 10.1016/ S0140-6736(18)30485-9

63. Ji L, Xie W, Zhang Z. Efficacy and Safety of Sirolimus in Patients With Systemic Lupus Erythematosus: A Systematic Review and Meta-Analysis. Semin Arthritis Rheum (2020) 50(5):1073-80. doi: 10.1016/ j.semarthrit.2020.07.006

64. Gerriets VA, Rathmell JC. Metabolic Pathways in T Cell Fate and Function. Trends Immunol (2012) 33(4):168-73. doi: 10.1016/j.it.2012.01.010

65. Bernatsky S, Boivin J-F, Joseph L, Manzi S, Ginzler E, Gladman DD, et al. Mortality in Systemic Lupus Erythematosus. Arthritis Rheumatism (2006) 54 (8):2550-7. doi: 10.1002/art.21955 
66. Szabo MZ, Szodoray P, Kiss E. Dyslipidemia in Systemic Lupus Erythematosus. Immunol Res (2017) 65(2):543-50. doi: 10.1007/s12026016-8892-9

67. Hahn BH, Grossman J, Chen WL, McMahon M. The Pathogenesis of Atherosclerosis in Autoimmune Rheumatic Diseases: Roles of Inflammation and Dyslipidemia. J Autoimmun (2007) 28(2-3):69-75. doi: 10.1016/ j.jaut.2007.02.004

68. McMahon M, Grossman J, Skaggs B, FitzGerald J, Sahakian L, Ragavendra N, et al. Dysfunctional Proinflammatory High-Density Lipoproteins Confer Increased Risk of Atherosclerosis in Women With Systemic Lupus Erythematosus. Arthritis Rheumatism (2009) 60(8):2428-37. doi: 10.1002/ art. 24677

69. Ardoin S, Sandborg C, Schanberg L. Review: Management of Dyslipidemia in Children and Adolescents With Systemic Lupus Erythematosus. Lupus (2007) 16(8):618-26. doi: 10.1177/0961203307079566

70. Nuttall SL, Heaton S, Piper MK, Martin U, Gordon C. Cardiovascular Risk in Systemic Lupus Erythematosus - Evidence of Increased Oxidative Stress and Dyslipidaemia. Rheumatology (2003) 42(6):758-62. doi: 10.1093/ rheumatology/keg212

71. Ryu H, Kim J, Kim D, Lee JE, Chung Y. Cellular and Molecular Links Between Autoimmunity and Lipid Metabolism. Mol Cells (2019) 42(11):747-54. doi: 10.14348/molcells.2019.0196

72. Yu X-H, Fu Y-C, Zhang D-W, Yin K, Tang C-K. Foam Cells in Atherosclerosis. Clin Chim Acta (2013) 424:245-52. doi: 10.1016/j.cca.2013.06.006

73. Kiss M, Czimmerer Z, Nagy L. The Role of Lipid-Activated Nuclear Receptors in Shaping Macrophage and Dendritic Cell Function: From Physiology to Pathology. J Allergy Clin Immunol (2013) 132(2):264-86. doi: 10.1016/ j.jaci.2013.05.044

74. Ryu H, Lim H, Choi G, Park YJ, Cho M, Na H, et al. Atherogenic Dyslipidemia Promotes Autoimmune Follicular Helper T Cell Responses via IL-27. Nat Immunol (2018) 19(6):583-+. doi: 10.1038/s41590-018-0102-6

75. Lim H, Kim YU, Sun H, Lee JH, Reynolds JM, Hanabuchi S, et al. Proatherogenic Conditions Promote Autoimmune T Helper 17 Cell Responses In Vivo. Immunity (2014) 40(1):153-65. doi: 10.1016/j.immuni.2013.11.021

76. Frostegard J, Wu RH, Giscombe R, Holm G, Lefvert AK, Nilsson J. Induction Of T-Cell Activation By Oxidized Low-Density-Lipoprotein. Arterioscler Thromb (1992) 12(4):461-7. doi: 10.1161/01.ATV.12.4.461

77. Dimeloe S, Burgener AV, Grahlert J, Hess C. T-Cell Metabolism Governing Activation, Proliferation and Differentiation; a Modular View. Immunology (2017) 150(1):35-44. doi: 10.1111/imm.12655

78. Simons K. Cell Membranes: A Subjective Perspective. BBA (2016) 1858 (10):2569-72. doi: 10.1016/j.bbamem.2016.01.023

79. Jury EC, Kabouridis PS, Flores-Borja F, Mageed RA, Isenberg DA. Altered Lipid Raft-Associated Signaling and Ganglioside Expression in $\mathrm{T}$ Lymphocytes From Patients With Systemic Lupus Erythematosus. J Clin Invest (2004) 113(8):1176-87. doi: 10.1172/JCI200420345

80. McDonald G, Deepak S, Miguel L, Hall CJ, Isenberg DA, Magee AI, et al. Normalizing Glycosphingolipids Restores Function in CD4(+) T Cells From Lupus Patients. J Clin Invest (2014) 124(2):712-24. doi: 10.1172/JCI69571

81. Surls J, Olsen C, Brumeanu T-D, Nazarov-Stoica C, Kehl M, Casares S. Increased Membrane Cholesterol in Lymphocytes Diverts T-Cells Toward an Inflammatory Response. Figshare (2012) 7(6):e38733. doi: 10.1371/ journal.pone.0038733

82. Robinson GA, Waddington KE, Pineda-Torra I, Jury EC. Transcriptional Regulation of T-Cell Lipid Metabolism: Implications for Plasma Membrane Lipid Rafts and T-Cell Function. Front Immunol (2017) 8:10. doi: 10.3389/ fimmu.2017.01636

83. Waddington KE, Papadaki A, Coelewij L, Adriani M, Nytrova P, Havrdova EK, et al. Using Serum Metabolomics to Predict Development of Anti-Drug
Antibodies in Multiple Sclerosis Patients Treated With IFN Beta. Front Immunol (2020) 11. doi: 10.3389/fimmu.2020.01527

84. Flores-Borja F, Kabouridis PS, Jury EC, Isenberg DA, Mageed RA. Altered Lipid Raft-Associated Proximal Signaling and Translocation of CD45 Tyrosine Phosphatase in B Lymphocytes From Patients With Systemic Lupus Erythematosus. Arthritis Rheum (2007) 56(1):291-302. doi: 10.1002/ art.22309

85. Waddington KE, Robinson GA, Rubio-Cuesta B, Chrifi-Alaoui E, Andreone S, Poon KS, et al. LXR Directly Regulates Glycosphingolipid Synthesis and Affects Human CD4+ T Cell Function. Proc Natl Acad Sci USA (2021) 118 (21). doi: 10.1073/pnas.2017394118

86. Heine G, Dahten A, Hilt K, Ernst D, Milovanovic M, Hartmann B, et al. Liver $\mathrm{X}$ Receptors Control IgE Expression in B Cells. J Immunol (2009) 182 (9):5276-82. doi: 10.4049/jimmunol.0801804

87. Cairoli E, Rebella M, Danese N, Garra V, Borba EF. Hydroxychloroquine Reduces Low-Density Lipoprotein Cholesterol Levels in Systemic Lupus Erythematosus: A Longitudinal Evaluation of the Lipid-Lowering Effect. Lupus (2012) 21(11):1178-82. doi: 10.1177/0961203312450084

88. Nossent J, Cikes N, Kiss E, Marchesoni A, Nassonova V, Mosca M, et al. Current Causes of Death in Systemic Lupus Erythematosus in Europe, 20002004: Relation to Disease Activity and Damage Accrual. Lupus (2007) 16 (5):309-17. doi: 10.1177/0961203307077987

89. Ruiz-Limon P, Barbarroja N, Perez-Sanchez C, Aguirre MA, Bertolaccini ML, Khamashta MA, et al. Atherosclerosis and Cardiovascular Disease in Systemic Lupus Erythematosus: Effects of In Vivo Statin Treatment. Ann Rheumatic Dis (2015) 74(7):1450-8. doi: 10.1136/annrheumdis-2013-204351

90. Petri MA, Kiani AN, Post W, Christopher-Stine L, Magder LS. Lupus Atherosclerosis Prevention Study (LAPS). Ann Rheumatic Dis (2011) 70 (5):760-5. doi: 10.1136/ard.2010.136762

91. Schanberg LE, Sandborg C, Barnhart HX, Ardoin SP, Yow E, Evans GW, et al. Use of Atorvastatin in Systemic Lupus Erythematosus in Children and Adolescents. Arthritis Rheumatism (2012) 64(1):285-96. doi: 10.1002/ art.30645

92. Yu H-H, Chen P-C, Yang Y-H, Wang L-C, Lee J-H, Lin Y-T, et al. Statin Reduces Mortality and Morbidity in Systemic Lupus Erythematosus Patients With Hyperlipidemia: A Nationwide Population-Based Cohort Study. Atherosclerosis (2015) 243(1):11-8. doi: 10.1016/j.atherosclerosis.2015.08.030

93. Jury EC, Isenberg DA, Mauri C, Ehrenstein MR. Atorvastatin Restores Lck Expression and Lipid Raft-Associated Signaling in T Cells From Patients With Systemic Lupus Erythematosus. J Immunol (2006) 177(10):7416-22. doi: 10.4049/jimmunol.177.10.7416

Conflict of Interest: The authors declare that the research was conducted in the absence of any commercial or financial relationships that could be construed as a potential conflict of interest.

Publisher's Note: All claims expressed in this article are solely those of the authors and do not necessarily represent those of their affiliated organizations, or those of the publisher, the editors and the reviewers. Any product that may be evaluated in this article, or claim that may be made by its manufacturer, is not guaranteed or endorsed by the publisher.

Copyright (c) 2022 Robinson, Wilkinson and Wincup. This is an open-access article distributed under the terms of the Creative Commons Attribution License (CC BY). The use, distribution or reproduction in other forums is permitted, provided the original author(s) and the copyright owner(s) are credited and that the original publication in this journal is cited, in accordance with accepted academic practice. No use, distribution or reproduction is permitted which does not comply with these terms. 